\title{
USO DO GRUPO OPERATIVO NA ATENÇÃO EM SAÚDE: REVISÃO INTEGRATIVA
}

Roselma Lucchese', Ivânia Vera², Patrícia Rosa Benicio ${ }^{3}$, Aline de Fátima da Silva ${ }^{4}$, Denize Bouttelet Munari ${ }^{5}$, Cinira Magali Fortuna ${ }^{5}$

${ }^{1}$ Enfermeira. Doutora em Enfermagem. Universidade Federal de Goiás. Catalão-GO-Brasil.

${ }^{2}$ Enfermeira. Doutora em Enfermagem. Universidade Federal de Goiás. Catalão-GO-Brasil.

${ }^{3}$ Discente de Graduação do Curso de Enfermagem. Universidade Federal de Goiás. Goiânia-GO-Brasil.

${ }^{4}$ Enfermeira. Doutora em Enfermagem. Universidade Federal de Goiás. Goiânia-GO-Brasil.

${ }^{5}$ Enfermeira.Doutora em Enfermagem. Universidade de São Paulo. Ribeirão Preto-SP-Brasil.

RESUMO: O objetivo deste estudo foi identificar como o Grupo Operativo tem sido utilizado na área de saúde e quais as evidências de seu uso na literatura científica. Foi realizada revisão integrativa, nas bases : Literatura Latino-Americana e do Caribe em Ciências da Saúde (LILACS), Medical Literature Analysis and Retrietal Systen On-Line (MEDLINE) e National Library of Medicine na base PUBMED. Selecionaram-se artigos publicados de 2002 a julho de 2014 que abordassem o Grupo Operativo proposto por Pichon-Rivière. Foram encontrados 23 artigos discutidos nas categorias : Grupo Operativo na Atenção à Saúde das Pessoas; Grupo Operativo no Processo Ensino-Aprendizagem do Profissional; Uso do Grupo Operativo na Gestão de Serviços em Saúde; Grupo Operativo na Pesquisa em Saúde. O Grupo Operativo é adotado na atenção à saúde em doenças crônicas, pesquisa, gestão e formação de trabalhadores de saúde. Conclui-se que essa ferramenta é relevante para o cuidado e que são necessárias pesquisas com outros delineamentos metodológicos.

DESCRITORES: Processos grupais; Saúde; Literatura de revisão como assunto; Assistência à saúde.

\section{USE OF THE OPERATIVE GROUP IN HEALTH CARE: AN INTEGRATIVE REVIEW}

\begin{abstract}
This study aimed to identify how the Operative Group has been used in the area of health, and what the evidence is for its use in the scientific literature. An integrative review was undertaken, in the following databases: Latin American and Caribbean Center on Health Science Information (LILACS), Medical Literature Analysis and Retrieval System On-Line (MEDLINE) and National Library of Medicine in the PUBMED database. Articles published between 2002 and July 2014, which addressed the Operative Group, as proposed by Pichon-Rivière, were selected. A total of 23 articles was found, discussed in the following categories: the Operative Group in Peoples' Health Care; the Operative Group in the Professional's Teaching-Learning Process; the Use of the Operative Group in the Management of Health Services; and, the Operative Group in Health Research. The Operative Group has been adopted in health care for chronic illnesses, research, management, and training of health workers. It is concluded that this tool is relevant for the care and that studies with other methodological designs are necessary. DESCRIPTORS: Group processes; Health; Review literature as a topic : Healthcare
\end{abstract}

\section{USO DEL GRUPO OPERATIVO EN LA ATENCIÓN EN SALUD: REVISIÓN INTEGRATIVA}

RESUMEN: El objetivo de este estudio fue identificar cómo el Grupo Operativo viene siendo utilizado en el área de salud y cuales las evidencias de su uso en la literatura científica. Fue realizada revisión integrativa, en las bases: Literatura Latinoamericana y de Caribe en Ciencias de la Salud (LILACS), Medical Literature Analysis and Retrietal Systen On-Line (MEDLINE) y National Library of Medicine en la base PUBMED. Fueron seleccionados artículos publicados de 2002 a julio de 2014 que abordasen el Grupo Operativo propuesto por Pichon-Rivière. Fueron hallados 23 artículos discutidos en las categorías: Grupo Operativo en la Atención a la Salud de las Personas; Grupo Operativo en el Proceso Enseñanza-Aprendizaje del Profesional; Uso del Grupo Operativo en la Gestión de Servicios en Salud; Grupo Operativo en la Investigación en Salud. El Grupo Operativo es adoptado en la atención a la salud en enfermedades crónicas, investigación, gestión y formación de trabajadores de salud. Se concluyse que esa herramienta es relevante para el cuidado y que son necesarias investigaciones con otros métodos. DESCRIPTORES: Procesos grupales; Salud; Literatura de revisión como asunto; Asistencia a la salud. 


\section{INTRODUÇÃO}

Em meio às demandas de saúde, atividades em grupo se configuram como tecnologia prevista no Sistema Nacional de Saúde ${ }^{(1)}$. Alguns pesquisadores as apontam como estratégias efetivas para promover saúde, estimular mudanças e melhorar a qualidade de vida das pessoas ${ }^{(2-4)}$.

Por sua aplicação ampla no campo da saúde, as atividades grupais podem constituir importante aliado do profissional de saúde, por possibilitarem, principalmente, o uso do potencial de ajuda das pessoas que convivem com problemas ou situações semelhantes ${ }^{(1,5)}$. Desse modo, é essencial o estudo acerca do tema, ponderado pela orientação de que o grupo operativo é um referencial teórico-técnico na constituição de uma prática realmente eficaz ${ }^{(6)}$.

No caso do presente estudo, elegeu-se como foco de interesse a técnica de Grupo Operativo (GO) proposta pelo psicanalista Pichón-Rivière. Os fundamentos do GO se baseiam na relação dinâmica e dialética entre sujeito e objeto, com a aprendizagem como fator terapêutico central. A aprendizagem é compreendida como um processo permanente que decorre da relação da pessoa com o outro e com o meio ${ }^{(7)}$. Para tal, o GO investe na leitura crítica da realidade e das relações, condições essenciais para atitudes investigadoras e mobilizadoras de mudança ${ }^{(5)}$.

A proposta do $\mathrm{GO}$ como referencial de grupo também possibilita a construção coletiva de projetos, fruto do diálogo e da escuta no espaço grupal, em busca da realização da tarefa comum entre os participantes ${ }^{(2)}$. Nessa perspectiva, o $\mathrm{GO}$ que, historicamente, foi projetado na área de saúde mental(7), vem ganhando espaço em outras áreas atenção à saúde das pessoas, na condução dos processos de mudança de hábitos de vida entre aqueles que convivem com doenças crônicas, por exemplo ${ }^{(4,8-9)}$.

Embora a literatura sinalize o uso do GO no contexto de ações de saúde, é fundamental conhecer em que aspectos ele tem sido mais comumente utilizado, quais resultados ele tem trazido e qual seu impacto nessas ações. Justifica-se, então, a relevância da análise da produção sobre o uso de GO na busca por proposição de práticas assertivas na área da saúde e na contribuição de investigações futuras.
Desse modo, o objetivo desta investigação foi identificar como o $\mathrm{GO}$ tem sido utilizado e quais as melhores evidências de seu uso produzidas na literatura científica.

\section{MÉTODO}

Para o estudo, utilizou-se a revisão integrativa (RI) como método de pesquisa, possibilitando a síntese da condição do conhecimento do estado da arte do assunto de interesse. A RI compreende seis etapas: identificação do tema, seleção da hipótese ou questão de pesquisa; estabelecimento de critérios para inclusão e exclusão de estudos/amostragem; definição das informações a serem extraídas dos estudos selecionados; avaliação dos estudos incluídos; interpretação dos resultados e apresentação da revisão/síntese do conhecimento ${ }^{(10-11)}$.

Considerando que o GO é uma ferramenta utilizada no processo de aprendizagem e terapêutico das pessoas, elaborou-se uma questão norteadora para a pesquisa: Quais evidências são produzidas com o uso da técnica do GO na atenção à saúde?

Para a realização da revisão, foram selecionadas as seguintes bases de dados: Literatura LatinoAmericana e do Caribe em Ciências da Saúde (LILACS), Medical Literature Analysis and Retrietal Systen On-Line (MEDLINE) e National Library of Medicine - National Institutes of Health (NLM$\mathrm{NIH})$ contendo a base de dados PUBMED. A pesquisa ocorreu entre os meses de julho e agosto de 2014, com coleta combinada em cada base de dados, por dois pesquisadores independentes.

Nas bases, utilizaram-se descritores controlados: "processos grupais OR group processes OR processos de grupo AND saúde OR health OR salud". Também se valeu de descritores não controlados: "grupo operativo AND saúde OR health OR salud", tendo em vista que o termo "Grupo Operativo" não consta da lista de descritores controlados Descritores em Ciências da Saúde (DeCS) da Biblioteca Virtual em Saúde (BVS)/Medical Subject Headings (MeSH), permitindo captura de maior número de artigos para a investigação.

Foram incluídos textos completos publicados entre 2002 e julho de 2014, em idioma inglês, espanhol e português. Os textos deveriam abordar a metodologia de trabalho GO tal como proposto por 
Pichon-Rivière ${ }^{(7)}$, com utilização de referenciais da literatura (acadêmica/científica) que subsidiassem a técnica e com sinalização de aplicação para um grupo específico, além de indicarem resultados práticos. Foram excluídos estudos teóricos de reflexão, atualização, revisão, tese e dissertação.
Visando aumentar a confiabilidade das informações nas bases de dados, foi realizada uma busca manual de artigos disponíveis (handsearching), orientada pelas referências das produções científicas já coletadas. Os resultados da seleção estão na Figura 1.
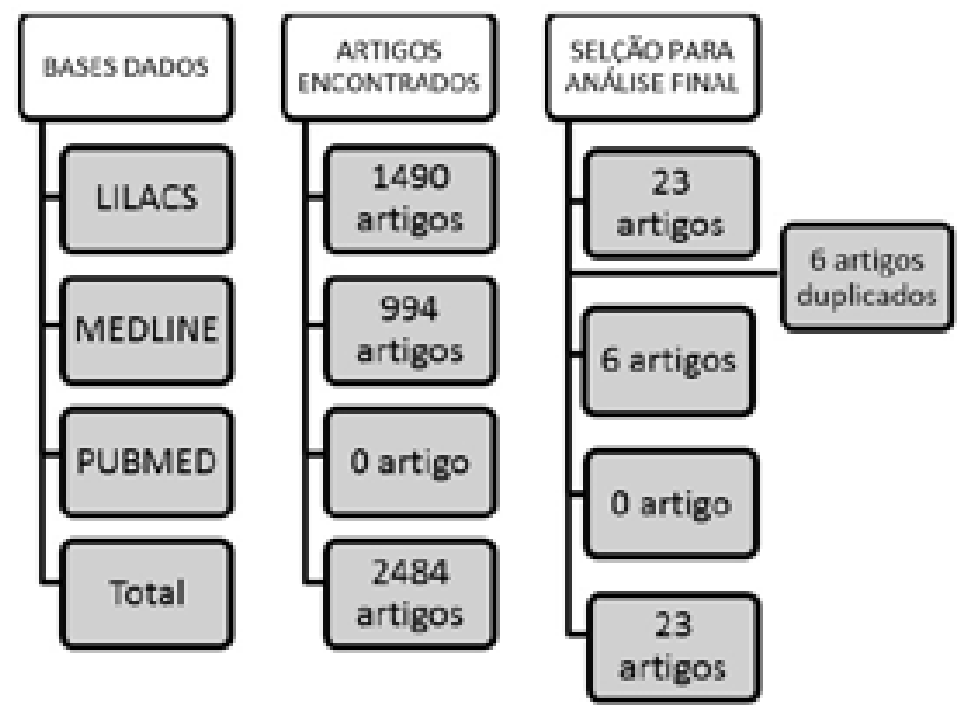

Figura 1 - Fluxograma da coleta de dados para Revisão Integrativa sobre Grupos Operativo na atenção à saúde. Goiás-Brasil, 2014

A análise dos dados derivados dos artigos incluídos foi mediada por um roteiro de coleta de dados focando o objetivo do estudo e os resultados encontrados. Também foi considerado, na análise dos estudos, o nível de evidência com classificação hierárquica proposta por Melnyk e Fineout-Overholt ${ }^{(12)}$ : nível I para evidência obtida do resultado de meta-análise de estudos clínicos controlados e com randomização; nível II para estudo randomizado, controlado e delineado; nível III para ensaios clínicos bem delineados sem randomização; nível IV para caso-controle ou estudo de coorte; nível $\vee$ para revisão sistemática e estudos descritivos e qualitativos; nível VI para evidências originadas de um único estudo descritivo ou qualitativo; nível VII para evidências baseadas em opiniões de especialistas ou com base em normas ou legislação.

\section{RESULTADOS}

A partir do processo de análise dos 23 artigos incluídos no estudo sistematizou-se a produção por meio de um quadro síntese (Quadro 1).

Em relação ao exposto no quadro 1, destacamse os anos de publicação dos artigos: 2010 (cinco), 2007 (quatro) e 2012 (quatro). Não houve publicação de artigos no primeiro semestre de 2014. A base de dados de maior prevalência foi a LILACS, evidenciando a produção latinoamericana acerca do tema. Houve publicações em revistas brasileira, mexicana e cubana, sobre as áreas de conhecimento no contexto da sáude. A área de maior prevalência foi a Enfermagem $(n=12)$, seguida da saúde coletiva (cinco).

Quanto à análise do nível de evidência, identificou-se maior número de artigos originais nível V ( $n=18)$, com apresentação de evidências de estudos qualitativos ou descritivos, com destaque especial para a metodologia pesquisaação ou interventiva. Do processo de análise das produções científicas, orientado pelo objetivo do estudo, emergiram quatro categorias: Grupo Operativo na Atenção à Saúde das Pessoas; Grupo Operativo no Processo Ensino-Aprendizagem do Profissional; Uso do Grupo Operativo na Gestão de Serviços em Saúde; Grupo Operativo na Pesquisa em Saúde. 


\begin{tabular}{|c|c|c|c|}
\hline Periódico/Ano/fonte & Fonte & Objetivo & Resultados com o GO \\
\hline Cardoso e Dall'Agnol(2) & $\begin{array}{l}\text { LILACS/ } \\
\text { MEDLINE }\end{array}$ & $\begin{array}{l}\text { Analyze the group process of } \\
\text { a nursing team at Hospital de } \\
\text { Clínicas de Porto Alegre }\end{array}$ & $\begin{array}{l}\text { Team learns and is able to identify } \\
\text { the main indicators of the group } \\
\text { process, it becomes possible to } \\
\text { improve operatively }\end{array}$ \\
\hline Grando e Dall'Agnol(3) & LILACS & $\begin{array}{l}\text { Analisar as reuniões de uma } \\
\text { equipe da Estratégia Saúde da } \\
\text { Família, mediante observação e } \\
\text { grupos focais }\end{array}$ & $\begin{array}{l}\text { Abordou a «equipe perfeita» do } \\
\text { imaginário da equipe e o que } \\
\text { dificulta o enfrentamento dos } \\
\text { conflitos }\end{array}$ \\
\hline Almeida e Soares(4) & $\begin{array}{l}\text { LILACS/ } \\
\text { MEDLINE }\end{array}$ & $\begin{array}{l}\text { Abordar o GO como espaço de } \\
\text { aprendizagem e transformação, e } \\
\text { refletir sobre a aprendizagem da } \\
\text { pessoa diabética }\end{array}$ & $\begin{array}{l}\text { Conquista de mais autonomia no } \\
\text { cuidado e manejo do diabetes } \\
\text { com a mudança de hábitos }\end{array}$ \\
\hline Soares e Ferraz $(8)$ & LILACS & $\begin{array}{l}\text { Analisar a aplicação da teoria e da } \\
\text { metodologia dos GO em grupos } \\
\text { de portadores de diabetes mellitus }\end{array}$ & $\begin{array}{l}\text { GO: melhor enfrentamento de } \\
\text { questões afetivas e a aprendizagem } \\
\text { das dimensões da doença }\end{array}$ \\
\hline Torres et al.(9) & $\begin{array}{l}\text { LILACS/ } \\
\text { MEDLINE }\end{array}$ & $\begin{array}{l}\text { Estimular o indivíduo a refletir } \\
\text { sobre seu estilo de vida cotidiano } \\
\text { relacionado ao diabetes }\end{array}$ & $\begin{array}{l}\text { Estimulou a relação interpessoal } \\
\text { entre os diabéticos e profissionais } \\
\text { de saúde } \\
\text { Troca de informações e adoção de } \\
\text { práticas saudáveis de saúde }\end{array}$ \\
\hline Lucchese et al.(14) & $\begin{array}{l}\text { LILACS/ } \\
\text { MEDLINE }\end{array}$ & $\begin{array}{l}\text { Discutir o uso da tecnologia } \\
\text { de GO como estratégia de } \\
\text { enfrentamento do uso do tabaco } \\
\text { dentro do Programa de Controle } \\
\text { do Tabagismo }\end{array}$ & $\begin{array}{l}\text { Mudança de comportamento, } \\
\text { melhor adesão ao tratamento }\end{array}$ \\
\hline Cassol et al.(15) & $\begin{array}{l}\text { LILACS/ } \\
\text { MEDLINE }\end{array}$ & $\begin{array}{l}\text { Descrever a percepção de usuários } \\
\text { de álcool e outras drogas acerca } \\
\text { de seu tratamento em um GO } \\
\text { voltado ao ensino-aprendizagem } \\
\text { em saúde }\end{array}$ & $\begin{array}{l}\text { Suporte para manter-se em } \\
\text { abstinência, espaço para o diálogo, } \\
\text { aprendizado e como responsável } \\
\text { pelas vitórias conseguidas na luta } \\
\text { contra o consumo de drogas }\end{array}$ \\
\hline Pedroni et al.(16) & LILACS & $\begin{array}{l}\text { Caracterizar a assistência da } \\
\text { equipe de Enfermagem à pessoa } \\
\text { idosa com hipertensão arterial }\end{array}$ & Mudança de hábitos alimentares \\
\hline Favoreto e Cabral(17) & LILACS & $\begin{array}{l}\text { Observar se as pessoas, com } \\
\text { base em uma vivência dialógica } \\
\text { ampliam os significados no } \\
\text { processo saúde-doença }\end{array}$ & $\begin{array}{l}\text { Promoção do diálogo e reflexão } \\
\text { com integração de aspectos } \\
\text { sociais e culturais de suas vidas e } \\
\text { adoecimento }\end{array}$ \\
\hline Botelho et al.(18) & LILACS & $\begin{array}{l}\text { Avaliar os aprendizados e } \\
\text { percepções dos escolares } \\
\text { participantes de um GO que visou à } \\
\text { promoção da alimentação saudável }\end{array}$ & $\begin{array}{l}\text { Respostas positivas em relação às } \\
\text { atividades desenvolvidas e fixação } \\
\text { das intervenções por parte das } \\
\text { crianças, mesmo findado o GO }\end{array}$ \\
\hline Figueroa et al.(19) & LILACS & $\begin{array}{l}\text { Realizar una adecuada lectura de lo } \\
\text { queocurre en un grupo comunitario } \\
\text { durante la realización de un } \\
\text { proyecto educativo nutricional }\end{array}$ & $\begin{array}{l}\text { Desarrolla una dinámica } \\
\text { participativa e abandono de la } \\
\text { visión individual, y comienza del } \\
\text { enfoque colectivo de trabajo }\end{array}$ \\
\hline Murakami et al.(20) & LILACS & $\begin{array}{llr}\text { Descrever e } & \text { analisar } & \text { uma } \\
\text { intervenção } & \text { grupal } & \text { sobre } \\
\text { sexualidade } & \text { e DST/Aids junto a } \\
\text { adolescentes } & \text { do sexo feminino }\end{array}$ & $\begin{array}{l}\text { Melhor articulação entre o pensar, } \\
\text { o sentir e o agir para um adolescer } \\
\text { saudável em relação à sexualidade } \\
\text { e à prevenção das DST }\end{array}$ \\
\hline Colosio et al.(21) & $\begin{array}{l}\text { LILACS/ } \\
\text { MEDLINE }\end{array}$ & $\begin{array}{l}\text { Investigar os efeitos da adoção de } \\
\text { um procedimento de intervenção } \\
\text { em GO no trabalho de prevenção } \\
\text { de infecção pelo HIV }\end{array}$ & $\begin{array}{l}\text { Grupo de intervenção: redução da } \\
\text { prática de sexo anal desprotegida; } \\
\text { aumento do número de respostas } \\
\text { favoráveis à prevenção. Hábito } \\
\text { sexual mais seguro }\end{array}$ \\
\hline
\end{tabular}




\begin{tabular}{|c|c|c|c|}
\hline Peres e Figueiredo(22) & LILACS & \begin{tabular}{|l|} 
Identificar as consequências \\
do trabalho realizado pelo GO \\
junto aos clientes que usavam \\
neurolépticos de ação prolongada \\
\end{tabular} & $\begin{array}{l}\text { Participação ativa do cliente } \\
\text { psiquiátrico; estabelecimento de } \\
\text { vínculos e melhora no tratamento }\end{array}$ \\
\hline Gatti e Andréa(23) & LILACS & $\begin{array}{l}\text { Refletir sobre o trabalho com um } \\
\text { GO com mães adolescentes em } \\
\text { risco de exploração sexual }\end{array}$ & $\begin{array}{l}\text { Restaurar em parte a autoconfiança } \\
\text { e a esperança para enfrentarem as } \\
\text { transformações da vida e o dia a } \\
\text { dia }\end{array}$ \\
\hline Macêdo e Andrade(24) & LILACS & $\begin{array}{l}\text { Analisar a participação de crianças } \\
\text { em oficinas da Ação Griô }\end{array}$ & $\begin{array}{l}\text { As oficinas favoreceram o } \\
\text { autocuidado e a descoberta de } \\
\text { habilidades, além da construção } \\
\text { da subjetividade }\end{array}$ \\
\hline Hur et al.(25) & LILACS & $\begin{array}{l}\text { Apresentar e discutir a experiência } \\
\text { de um curso chamado Psicanálise } \\
\text { de grupos: teoria e técnica }\end{array}$ & $\begin{array}{l}\text { Mudança significativa no que se } \\
\text { refere à diminuição de ansiedades } \\
\text { e à implicação para a elaboração } \\
\text { da aprendizagem }\end{array}$ \\
\hline Lucchese e Barros(26) & $\begin{array}{l}\text { LILACS/ } \\
\text { MEDLINE }\end{array}$ & \begin{tabular}{|l|} 
Oferecer um espaço para a \\
expressão vivencial do aluno de \\
Enfermagem
\end{tabular} & $\begin{array}{l}\text { Espaço continente das vivências } \\
\text { dos alunos em seu processo de } \\
\text { formação }\end{array}$ \\
\hline Correa et al.(27) & LILACS & \begin{tabular}{|l|l|} 
Analisar a utilização GO \\
como espaço para a troca de \\
experiências entre os discentes de \\
Enfermagem \\
\end{tabular} & $\begin{array}{l}\text { Efetivação de troca de } \\
\text { experiências entre os discentes no } \\
\text { enfrentamento da transição para a } \\
\text { vida profissional } \\
\end{array}$ \\
\hline Oliveira e Ciampone(28) & LILACS & $\begin{array}{l}\text { Identificar as percepções que os } \\
\text { alunos de Enfermagem têm em } \\
\text { relação à qualidade de vida }\end{array}$ & $\begin{array}{l}\text { Situações dialéticas em relação à } \\
\text { promoção da qualidade de vida } \\
\text { dos alunos promoveu a qualidade } \\
\text { de vida dos alunos }\end{array}$ \\
\hline Gama e Koda(30) & LILACS & $\begin{array}{l}\text { Promover intervenção e análise } \\
\text { psicológica comunitária em uma } \\
\text { cooperativa de reciclagem de } \\
\text { resíduos sólidos }\end{array}$ & $\begin{array}{l}\text { GO oportunizou maior } \\
\text { participação de seus membros e } \\
\text { construção da figura de agente } \\
\text { ambiental em substituição à } \\
\text { identificação com lixo }\end{array}$ \\
\hline Böck et al.(32) & LILACS & $\begin{array}{l}\text { Concretizar e sistematizar suporte } \\
\text { social e afetivo para os professores, } \\
\text { considerando a proposta de } \\
\text { remissão de burnout }\end{array}$ & $\begin{array}{l}\text { Aumento do nível de Burnout, } \\
\text { nas dimensões: esgotamento } \\
\text { emocional, realização profissional } \\
\text { e despersonalização no grupo } \\
\text { experimental. Melhora nas } \\
\text { estratégias de enfrentamento para } \\
\text { o esgotamento profissional } \\
\end{array}$ \\
\hline Pereira et al.(33) & LILACS & $\begin{array}{l}\text { Relatar a experiência de } \text { (re) } \\
\text { construção de um grupo de } \\
\text { planejamento familiar numa } \\
\text { Unidade Básica de Saúde }\end{array}$ & 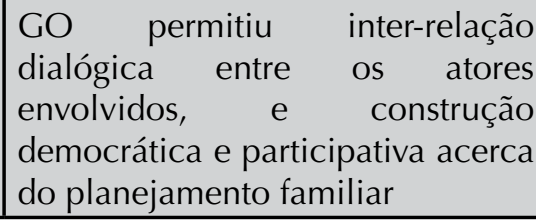 \\
\hline
\end{tabular}




\section{DISCUSSÃO}

Entre as produções estudadas, destaca-se a utilização do GO na prática de Enfermagem, com maior produção científica identificada nesta RI. Tal fato que pode ser explicado pela identificação desses profissionais com o pressuposto pichoniano, além da relevante ferramenta para o aprimoramento do cuidado. O crescente interesse pela temática de grupos na Enfermagem também ocorre por sua aplicação como estratégia de liderança do cuidado ao ser humano, no ensino e na pesquisa, dentre outros ${ }^{(2,8)}$. Dicute-se que o desenvolvimento de tecnologia de grupo, na atenção de pessoas, contribui inclusive para o próprio amadurecimento do enfermeiro ${ }^{(13)}$.

A área de saúde coletiva também representou um campo significativo na divulgação de produção de conhecimento por meio do GO, com a participação de psicólogos, enfermeiros, nutricionistas, médicos e educadores. Talvez essa seja uma resposta às demandas da área, pois é nesse contexto em que ações coletivas são mais exigidas e imprimem ao profissional de saúde um «fazer grupos» constante, seja ao atender as complexidades de gestão ou de necessidades de saúde ${ }^{(1)}$. Para essa última, o uso do GO proporcionou intervenção considerando a relação dialética do indivíduo com o meio em que vive, em um ambiente coletivo, potencializando, assim, uma mudança de hábitos e promoção da saúde ${ }^{(2)}$.

Em relação à multiplicidade do uso do GO na área da saúde das pessoas, verifica-se a diversidade dos cenários em que essa técnica foi aplicada. Entre estes, incluem-se os hospitais, ambulatórios, Atenção Primária à Saúde, ambientes de trabalho, e instituições de ensino infantil e superior.

Quanto ao nível de evidência ${ }^{(12)}$ dos artigos incluídos, os resultados com predomínio de nível V, isto é, estudos descritivos e qualitativos, indicaram ser fundamental o desenvolvimento de projetos com delineamentos metodológicos mais robustos para o estudo das intervenções grupais em particular, aqueles que privilegiem a avaliação sistematizada do impacto dessas intervenções.

\section{Grupo Operativo na Atenção à Saúde das Pessoas}

Em relação aos cuidados da saúde das pessoas, o GO foi aplicado com a finalidade de promover a aprendizagem, identificando dificuldades, discutindo possibilidades e encontrando soluções adequadas para o problema latente em sua condição de vida ${ }^{(14-15)}$. Em questão aos usuários participantes do GO, a tarefa do grupo é aprender a conviver com a doença crônica, como o diabetes mellitus e a hipertensão arterial sistêmica (HAS); desenvolver autonomia e autocuidado; e aderir ao tratamento ${ }^{(4,9,16)}$, no enfrentamento do uso de tabaco, álcool e outras drogas, por exemplo ${ }^{(14-15)}$. Para tanto, o GO pode ser articulado a jogos educativos que intensificam trocas de saberes ${ }^{(9)}$.

A utilização das atividades grupais no cuidado às pessoas favorece a efetividade dos objetivos quanto ao cuidado de saúde ${ }^{(17)}$, como hábitos saudáveis de alimentação ${ }^{(18)}$. Nesse sentido, o trabalho em conjunto proporciona troca de ideias e experiências entre os participantes, especialmente com a utilização de estratégias dinâmicas. Nesse ínterim, a escola é um local apropriado para a realização do GO com criança, por se tratar de ambiente específico de ensino ${ }^{(18)}$.

O GO também se sincroniza com metodologia participativa na construção de um projeto nutricional coletivo. Com o propósito de educação em saúde, necessita-se de atividades das quais a comunidade participe, sendo capaz de identificar os problemas, formular ações preventivas, executar tarefas e avaliar os objetivos alcançados. O propósito é a corresponsabilidade em relação aos problemas, favorecendo o processo educacional no grupo, por meio da exploração do modo de ser, da realidade vivenciada, das incertezas, dos pensamentos e das expectativas ${ }^{(19)}$.

Dentre os grupos específicos nos quais foram utilizados o GO, destaca-se o grupo de adolescentes na abordagem do planejamento familiar, promoção da sáude e prevenção de Doenças Sexualmente Transmissíveis (DST)(20), bem como na prevenção do HIV/Aids entre homens que fazem sexo com homens ${ }^{(21)}$. Em ambos os estudos, foi ampliada a visão acerca do tema e foram encontradas respostas mais efetivas para prevenir infecções.

$\mathrm{Na}$ área de especialidade de Psiquiatria, o GO revelou-se um importante instrumento de cuidado aos pacientes que usam neurolépticos. Ao se abordar a dimensão do cotidiano das pessoas, o GO oportuniza um momento de fala e de escuta, com intercâmbios afetivos e trocas sociais que 
privilegiam a tarefa grupal ${ }^{(22)}$. Também em relação à promoção da saúde mental, o GO revelou-se um meio de enfrentamento de situações de risco de população vulnerável, em especial à violência e à probreza ${ }^{(23-24)}$.

\section{Grupo Operativo no Processo Ensino- Aprendizagem do Profissional}

Por meio do GO, há possibilidades dos alunos e educadores desenvolverem capacidades de crítica, diálogo e compreensão para amadurecimento pessoal e grupal| ${ }^{(25-26)}$ condições estas que favorecem o enfrentamento de situações de apropriação de uma profissão, na finalização da graduação em Enfermagem ${ }^{(26-27)}$.

Em outro estudo sobre o processo ensinoaprendizagem do aluno de graduação em Enfermagem, o GO demonstrou-se continente dos sentimentos despertados no aprendiz durante a formação. Em alguns casos, essa afetividade mobilizada é de angústia, medo e insegurança diante do sobrecarga da prática de uma profissão que lida com a vida, o que pode trazer consequências negativas ao futuro profissional, se não houver dispositivos para esse acolhimento. $\mathrm{O} \mathrm{GO}$ foi um instrumento assertivo para tais momentos ${ }^{(26-28)}$.

Uma das propostas do GO é que o sujeito amplie sua visão de coletividade, inicie com a pertença a um determinado grupo e amplie a compreensão da inserção das pessoas em grupo. A sala de aula mostrou-se um ambiente propício ao aprendizado de vários sujeitos, especialmente na graduação em Enfermagem, tendo em vista que estes se tornem futuros profissionais críticos, autônomos e cidadãos ${ }^{(3,28)}$.

\section{Uso do Grupo Operativo na Gestão de Serviços em Saúde}

O conhecimento da tecnologia de GO também foi aplicado na leitura da dinâmica de funcionamento de equipes de saúde. Da compreensão de trabalho em grupo, com suas relações cotidianas e seus conflitos, emergiram questões desafiadoras, como o compartilhar de um objetivo comum para as ações coletivas e o desenvolvimento do respeito mútuo. Tais questões estão evidentes nos trabalhadores de Enfermagem que, por natureza, atuam em grupo. Assim, as concepções de grupo deveriam permear sua vida profissional(2).

A análise de equipes, como a do Programa de Saúde da Família, à luz pichoniana, permitiu a abordagem de sua pretensão essencial que é a de inovar a forma de atenção à saúde dos indivíduos, com a missão de superar ações não resolutivas, como receber prescrições de remédios e recomendações de mudanças de hábitos. Para tanto, considerou-se respeitável que a equipe fosse treinada por meio das técnicas do GO, permitindo transformações e desenvolvimento, pois, no grupo, os membros da equipe pensam conjuntamente sobre os aspectos que necessitem de melhoras, seja na forma como trabalham ou como se relacionam ${ }^{(29)}$.

O saber produzido com a aplicação do GO igualmente possibilitou a análise da Equipe Saúde da Família que se organizou em torno de uma «equipe perfeita» idealizada, em que se evitam as discussões polêmicas quando o conflito é percebido como ameaçador à sua integridade. A leitura dessa realidade, por meio do GO, apontou a fragilidade da equipe em gerenciar conflitos e também revelou sua potencialidade na elaboração de projetos, frente aos problemas cotidianos $^{(3)}$. Percebe-se, então, a virtuosidade do GO na gestão de equipes.

Além disso, o trabalho de GO abordou profissionais de uma cooperativa de reciclagem de resíduos sólidos e proporcionou interação entre os participantes, com reforço para confiança em um ambiente antes permeado pela desconfiança. Desse modo, o grupo serviu para que os integrantes superassem resistências em relação aos companheiros de trabalho, pois todos tiveram direito de voz durante as reuniões grupais ${ }^{(30)}$.

\section{Grupo Operativo na Pesquisa em Saúde}

Pela produção de conhecimento da RI, observa-se que o grupo foi aplicado nas pesquisas com finalidades distintas, como intervenção junto à população de estudo ${ }^{(30-33)}$, como técnica de obtenção de dados empíricos ${ }^{(28)}$ ou na leitura e condução da análise de uma dada realidade(3). Nesse sentido, a aplicação do GO, na pesquisa na saúde, conduz o estudo desde a coleta de dados até a análise deles, contanto com referencial teórico para inferência e discussão. O GO pode 
subsidiar a intepretação da realidade, além de contar com dispositivos que lhe são inerentes, como o registro das crônicas, a mediação da dinâmica do grupo com o coordenador e observador, os tempos grupais com devolutiva, abertura, desenvolvimento e encerramento ${ }^{(31)}$.

\section{CONSIDERAÇÕES FINAIS}

O GO vem sendo utilizado e indicado na área da saúde com uma produção científica relevante em pouco mais de uma década de busca. Os enfermeiros foram os profissionais que mais publicaram, confirmando a identidade que a profissão tem com a tecnologia e o interesse em se apropriar de referencial que subsidie o cuidado. Quanto à população trabalhada nos estudos, houve uma diversidade com relação a: fases do ciclo de vida, gênero, orientação sexual, agrupamentos específicos conforme patologia, trabalhadores da Enfermagem (saúde), estudante de graduação na saúde e educadores de alunos de graduação.

Ao mesmo tempo, a utilização do GO na educação em saúde se destacou nesta RI. Foi frequente sua associação com referenciais didático-pedagógicos em processos de ensinoaprendizagem, como o construtivismo, ou em práticas didáticas interativas, própria das pedagogias ativas.

A compreensão e as intervenções nas relações de trabalho por meio do GO, seja em equipe de saúde ou por profissionais de saúde, possibilitaram a abordagem de dificuldades, apontando para fatores conflituosos a serem gerenciados. Essas ações oportunizaram a elaboração de projetos, compreendidos como salto qualitativo na dinâmica das equipes, evidenciando o uso do GO na gestão com pessoas.

Sem dúvida, as evidências dos estudos analisados afirmam a característica do GO como a um grupo de aprendizagem. No entanto, elas apontam para os ganhos terapêuticos que se alcançam por meio dessa tecnologia, quer na educação e formação de profissionais, quer na atenção à saúde das pessoas.

É importante ressaltar que os artigos incluídos nesta RI corroboram a utilização do GO em possibilidades diversas de pesquisa. E, o fato de os estudos analisados serem, em sua maioria, pesquisas de cunho qualitativo e descritivo, sugere estudos com delineamentos metodológicos de melhor classificação hierárquica, segundo níveis de evidência. Contudo, as pesquisas estudadas abordaram um objeto de estudo complexo e promissor, constiutindo fontes de conhecimento significativas para pesquisas futuras. Como limite da RI, coloca-se a não indexação do termo "Grupo Operativo" em bases de dados científicos, o que impediu uma busca direta da produção.

\section{REFERÊNCIAS}

1. Munari DB, Lucchese R, Medeiros M. Reflexão sobre o uso de atividades grupais na atenção a portadores de doenças crônicas. Cienc. cuid. saude. [Internet] 2009;(8) [acesso em 27 ago 2014]. Disponível: http:// periodicos.uem.br/ojs/index.php/CiencCuidSaude/ article/viewFile/9742/5545

2. Cardoso ASF, Dall'agnol CM. Processo grupal: reflexões de uma equipe de enfermagem. Rev Esc Enferm USP. 2011;45(6):1406-12.

3. Grando MK, Dall'agnol CM. Desafios do processo grupal em reuniões de equipe da estratégia saúde da família. Esc. Anna Nery. [Internet] 2010;14(3) [acesso em 29 ago 2014]. Disponível: http://dx.doi.org/10.1590/ S1414-81452010000300011

4. Almeida SP, Soares SM. Aprendizagem em grupo operativo de diabetes uma abordagem etnográfica. Ciênc. saúde colet. [Internet] 2010;15(1) [acesso em 29 ago 2014]. Disponível: http://dx.doi.org/10.1590/ S1413-81232010000700020

5. Bastos ABBI. A técnica de grupos-operativos à luz de Pichon-Rivière e Henri Wallon. Psicol. inf. [Internet] 2010;14(14) [acesso em 27 ago 2014]. Disponível: https://www.metodista.br/revistas/revistasims/index. php/PINFOR/article/viewFile/2348/2334

6. Simões FV, Stipp AC. Grupos na enfermagem: classificação, terminologias e formas de abordagem. Esc. Anna Nery. [Internet] 2006;10(1) [acesso em 29 ago 2014]. Disponível: http://dx.doi.org/10.1590/ S1414-81452006000100019

7. Pichon-Rivière. E. Processo Grupal. $8^{\mathrm{a}}$ ed. São Paulo: WMF Martins Fontes; 2009.

8. Soares SM, Ferraz AF. Grupos operativos de aprendizagem nos serviços de saúde, sistematização de fundamentos e metodologia. Esc. Anna Nery. [Internet] 2007;11(1) [acesso em 29 ago 2014]. Disponível: http:// dx.doi.org/10.1590/S1414-81452007000100007

9. Torres HC, Hortale VA, Schall V. A experiência de jogos em grupos operativos na educação em saúde para diabéticos. Cad. Saúde Pública. [Internet] 
2003;19(4) [acesso em 29 ago 2014]. Disponível: http:// dx.doi.org/10.1590/S0102-311X2003000400026

10. Mendes KDS, Silveira RCCP, Galvão CM. Revisão integrativa: método de pesquisa para a incorporação de evidências na saúde e na enfermagem. Texto Contexto Enferm. [Internet] 2008;17(4) [acesso em 29 ago 2014]. Disponível: http://dx.doi.org/10.1590/ S0104-07072008000400018

11. Ganong LH. Integrative reviews of nursing research. Res Nurs Health. 1987;10(1):1-11.

12. Melnyk BM, Fineout-Overholt E. Making the case for evidence-based practice. In: Melnyk BM, FineoutOverholt E. Evidence-based practice in nursing \& healthcare. A guide to best practice. Philadelphia: Lippincot Williams \& Wilkins; 2005. p. 3-24.

13. Vasconcelos SC, Frazão IS, Ramos VP. Grupo terapêutico educação em saúde: subsídios para a promoção do autocuidado de usuários de substâncias psicoativas. Cogitare enferm. 2012;17(3):498-505.

14. Lucchese R, Vargas LS, Teodoro WR, Santana LKB, Santana FR. A tecnologia de grupo operativo aplicada num programa de controle do tabagismo. Texto Contexto Enferm. [Internet] 2013;22(4) [acesso em 29 ago 2014]. Disponível: http://dx.doi.org/10.1590/ S0104-07072013000400007

15. Cassol PB, Terra MG, Mostardeiro SCTS, Gonçalves MO, Pinheiro UMS. Tratamento em um grupo operativo em saúde: percepção dos usuários de álcoole outras drogas. Rev. Gaúcha Enferm. [Internet] 2012;33(1) [acesso em 27 ago 2014]. Disponível: http:// seer.ufrgs.br/index.php/RevistaGauchadeEnfermagem/ article/view/21664

16. Pedroni GAM, Rosa JA, Almeida MEF, Guedes HM. Assistência de enfermagem prestada à pessoa idosa com hipertensão arterial. R. Enferm. Cent. O. Min. [Internet] 2013;3(2) [acesso em 27 ago 2014]. Disponível: http://www.seer.ufsj.edu.br/index.php/ recom/article/viewFile/379/429

17. Favoreto CAO, Cabral CC. Narrativas sobre o processo saúde-doença: experiências em grupos operativos de educação em saúde. Interface - Comunic., Saúde, Educ. [Internet] 2009;13(28) [acesso em 29 ago 2014]. Disponível: http://dx.doi.org/10.1590/S141432832009000100002

18. Botelho LP, Zanirati VF, Paula DV, Lopes ACS, Santos LC. Promoção da alimentação saudável para escolares: aprendizados e percepções de um grupo operativo. Rev. Nutrire. 2010;35(2):103-16.

19. Figueroa IAV, Guerra JF, Gallegos NG. Proceso de grupo durante larealización de unproyecto educativo nutricional comunitário. Rev. Cub. Salud Publica. 2010;36(2):148-55.
20. Murakami JK, Petrilli Filho JF, Telles Filho PCP, Acorinte AC, Napoleão AA. Planejando, desenvolvendo e avaliando uma intervenção grupal junto a adolescentes: uma perspectiva sistêmica. Rev. Eletr. Enf. [Internet] 2007;3(9) [acesso em 27 ago 2014]. Disponível: http:// www.fen.ufg.br/revista/v9/n3/v9n3a16.htm

21. Colosio R, Fernandes MIA, Bergamaschi DP, Scarcelli IR, Lopes IC, Hearst N. Prevenção de infecção pelo HIV por intermédio da utilização do grupo operativo entre homens que fazem sexo com homens, São Paulo, Brasil. Cad. Saúde Pública. [Internet] 2007;23(4) [acesso em 27 ago 2014]. Disponível: http://dx.doi. org/10.1590/S0102-311X2007000400022

22. Peres MAA, Figueiredo NMA. Grupo do NAP: atendimento ambulatorial ao cliente em uso de medicação depósito - uma ação diferenciada de cuidar em psiquiatria. Texto Contexto Enferm. [Internet] 2004;4(13) [acesso em 27 ago 2014]. Disponível: http:// dx.doi.org/10.1590/S0104-07072004000400005

23. Gatti MC, Andréa MA. Acolhendo e ressignificando experiências de vida em grupo com mães adolescentes em risco de exploração sexual. Rev. SPAGESP. 2012;13(1):54-61.

24. Macêdo CMV, Andrade RGNA. Imagem de si e autoestima: a construção da subjetividade no grupo operativo. Psicol. Pesq. UFJF. [Internet] 2012;6(01) [acesso em 27 ago 2014]. Disponível: http://www.ufjf. br/psicologiaempesquisa/files/2012/09/v6n1a10.pdf

25. Hur DU, Alencar AKS, Almeida HB. Experiências de formação com dispositivos psicanalíticos de grupo com não psicólogos. Rev. SPAGESP [Internet] 2012;13(2) [acesso em 27 ago 2014]. Disponível: http:// pepsic.bvsalud.org/pdf/rspagesp/v13n2/v13n2a09.pdf

26. Lucchese R, Barros S. Grupo operativo como estratégia pedagógica em um curso graduação em enfermagem: um continente para as vivências dos alunos quartanistas. R Rev Esc Enferm USP. [Internet] 2002;36(1) [acesso em 27 ago 2014]. Disponível: http:// dx.doi.org/10.1590/S0080-62342002000100010

27. Correa AK, Souza MCBM, Saeki T. Transição para o exercício profissional em enfermagem: uma experiência em grupo operativo. Esc. Anna Nery. [Internet] 2005;9(3) [acesso em 29 ago 2014]. Disponível: http://revistaenfermagem.eean.edu.br/ detalhe_artigo.asp?id=73

28. Oliveira RA, Ciampone MHT. Qualidade de vida de estudantes de enfermagem: a construção de um processo e intervenções. Rev Esc Enferm USP. 2008;42(1):57-65.

29. Fortuna CM, Mishima SM, Matumoto S, Pereira MJB. O trabalho de equipe no programa de saúde da família: reflexões a partir de conceitos do processo grupal e de grupos operativos. Rev. Latino-Am. 
Enfermagem. [Internet] 2005;13(2) [acesso em 27 ago 2014]. Disponível: http://dx.doi.org/10.1590/S010411692005000200020

30. Gama CAP, Koda MY. Cooperativismo e reciclagem de resíduos sólidos: uma reflexão a partir da psicanálise de grupos. Cad. Psicol. Soc. Trab. [Internet] 2010;13(2) [acesso em 27 ago 2014]. Disponível: http://www. revistas.usp.br/cpst/article/view/25726/27459

31. Lucchese R, Barros S. A utilização do grupo operativo como método de coleta de dados em pesquisa qualitativa. Rev. Eletr. Enf. [Internet] 2007;9(3) [acesso em 27 ago 2014]. Disponível: http://www.revistas.ufg. br/index.php/fen/article/view/7505/5323

32. Böck VR, Sarriera JC. O grupo operativo intervindo na síndrome de Burnout. Psicol. Esc. Educ. [Internet] 2006;10(1) [acesso em 29 ago 2014]. Disponível: http:// dx.doi.org/10.1590/S1413-85572006000100004

33. Pereira QLC, Silva CBDCA, Pelzer MT, Lunardi VL, Siqueira $\mathrm{HCH}$. Processo de (re)construção de um grupo de planejamento familiar: uma proposta de educação popular em saúde. Texto Contexto Enferm. [Internet] 2007;16(2) [acesso em 27 agosto 2014]. Disponível: http://dx.doi.org/10.1590/S0104-07072007000200016 